\title{
Methodologic approaches in studies using real-world data (RWD) to measure pediatric safety and effectiveness of vaccines administered to pregnant women: A scoping review
}

\author{
Tamar Lasky ${ }^{a,}{ }^{*}$, Ann W. McMahon ${ }^{\mathrm{a}}$, Wei Hua ${ }^{\mathrm{b}}$, Richard Forshee $^{\mathrm{c}}$ \\ aUS Food and Drug Administration, Office of the Commissioner, Silver Spring, Maryland, USA \\ bUS Food and Drug Administration, Center for Drugs Evaluation and Research, Silver Spring, \\ Maryland, USA \\ cUS Food and Drug Administration, Center for Biologics Evaluation and Research, Silver Spring, \\ Maryland, USA
}

\begin{abstract}
Objective: This scoping review mapped studies using real-world data (RWD) to measure pediatric safety and effectiveness of vaccines administered to pregnant women.
\end{abstract}

Introduction: In the US, two vaccines are recommended for all pregnant women to prevent illness in the infant: inactivated influenza vaccine (recommended since 2004), and the combined tetanus-diphtheria-acellular pertussis (Tdap) vaccine (recommended since 2013). This scoping review maps the studies conducted to date that address questions about pediatric safety and effectiveness of vaccines administered during pregnancy and provides a knowledge base for evaluating the use of RWD to study this issue.

Methods: The scoping review was conducted following a published protocol. Methods included an electronic search of PubMed and Embase, screening of titles and abstracts by two reviewers, and double extraction of data for summary and synthesis. Studies that reported on pregnant women and the effectiveness or safety outcomes in their infants were included.

Results: Forty-eight studies met the inclusion criteria of the scoping review protocol using RWD to assess safety or effectiveness of influenza or pertussis vaccinations administered to pregnant women with respect to pregnancy, infant or child outcomes. Detailed information about data sources, linkage of maternal and infant data, and operational definitions for gestational age were largely absent from the majority of studies raising concerns about reproducibility and validity of study findings.

Conclusions: A body of literature is available from which to plan and design future studies of vaccination in pregnant women using RWD. This is of intense importance as new vaccines, such

*Corresponding author. Tamar.Lasky@fda.hhs.gov (T. Lasky).

Declaration of Competing Interest

The authors declare that they have no known competing financial interests or personal relationships that could have appeared to influence the work reported in this paper. 
as those for COVID-19, become available to the general population via approval or authorization without inclusion of pregnant women in the clinical trials.

\section{Keywords}

Vaccination; Infant; Influenza; Pertussis; Pregnancy

\section{Introduction}

Maternal vaccination aims to prevent illness in pregnant women, and/or confer passive immunity to the fetus [1-6]. In the US, two vaccines are recommended for all pregnant women to prevent illness in the infant (as well as preventing illness in the mother, as with influenza vaccine): inactivated influenza vaccine (recommended since 2004), and the combined tetanus-diphtheria-acellular pertussis (Tdap) vaccine (recommended since 2013) [7-10]. When the mother is vaccinated during pregnancy, both of these vaccines may confer immunity and/or protection to the fetus, particularly between birth and when the first infant immunizations are given, when the risk of illness is highest for the infant $[1,3]$. Infants under six months of age can experience severe complications or death if they contract influenza, however influenza vaccine is not approved for infants younger than six months of age [11]. Similarly, infants have a high probability of hospitalization and severe complications if they contract pertussis, and cannot be vaccinated until they are two months of age [3,12]. While other vaccines may be administered to pregnant women for maternal protection, in the United States, no other vaccines are currently recommended to confer immunity to the infant and are thus outside the focus of this review [9].

The increased interest in the use of real-world data (RWD) to provide real-world evidence (RWE) to support regulatory decision-making for products, including vaccines, is stated in the FDA framework: "RWD are data relating to patient health status and/or the delivery of health care routinely collected from a variety of sources." [13] (p. 4) and "RWE is the clinical evidence about the usage and potential benefits or risks of a medical product derived from analysis of RWD”.[13] (p.4) Randomized controlled trials have been conducted to estimate efficacy of maternal immunization in preventing influenza and pertussis in infants, as have studies using RWD $[14,15]$. While such studies show benefits of maternal immunization in preventing infant illness and no evidence of severe adverse events, there is continued interest in assessing safety and effectiveness of each season's influenza vaccines, and in monitoring the population effects on infant pertussis. In addition, it is of interest to policy makers and regulators to describe potential effects on secondary outcomes such as birth-weight or hospitalization, and to describe the duration of benefit. In countries such as the US, where pertussis and influenza vaccines are recommended for use in pregnant women, ethical issues around randomization in the absence of equipoise may constrain the use of randomized trials, thus increasing the utility of alternative approaches such as RWE. This is consistent with an increased focus on regulatory use of RWE, and may offer advantages with regards to larger sample size, greater population heterogeneity, and lower study costs. The rapidly developing scenarios around authorization and use of COVID-19 vaccines has resulted in administration of vaccines without previous clinical trial evidence regarding safety and efficacy in pregnancy. This increases the imperative to optimize the use 
of RWD to study safety and effectiveness of COVID-19 vaccines in pregnancy. The recent launch of clinical trials enrolling pregnant women may alter the evidence landscape, which is dynamic, changing as we write.

Considerable efforts have been made to develop assessment tools to guide the use of RWD/RWE in a range of policy contexts. One example is the Good Research for Comparative Effectiveness (GRACE) guidelines developed for non-interventional studies of comparative effectiveness to determine which studies are sufficiently rigorous to be reliable enough for use in health technology assessments [16]. A second example is the Joint ISPOR-ISPE Special Task Force publication on good practices for RWD studies of treatment and/or comparative effectiveness [17]. More specifically, there have been efforts to set guidelines for database selection, and checklists to ensure regulatory grade data quality have also been published, as well as guidelines for the use of RWD in studying COVID-19 vaccines in pregnancy [18-20]. None of these efforts have been directed at the highly specialized and unique challenges of studying effectiveness and safety of maternal vaccines administered to prevent disease in the infant.

This scoping review mapped studies using RWD/RWE to measure pediatric (encompassing fetal, newborn, and pediatric outcomes and distinguished from effects occurring to the mother) safety and effectiveness of vaccines administered to the mother during pregnancy. This work will help clarify approaches to key methodologic issues such as documentation of the vaccine administered, linkage of maternal and infant data, estimation of gestational age, and definition of safety and effectiveness outcomes.

\section{Methods}

The scoping review was conducted in accordance with the JBI methodology for scoping reviews [21,22]. It followed the Preferred Reporting Items for Systematic Review and MetaAnalysis Protocols Extension for Scoping Reviews (PRISMA-ScR) methodology [21-24]. It aimed to address the following questions:

i. What studies using RWD have been conducted to measure pediatric safety and effectiveness of influenza or pertussis vaccines administered to the mother during pregnancy?

ii. What electronic healthcare, administrative claims, or patient registry databases have been used in the above studies?

iii. What methods were used to link maternal and infantrecords?

iv. How did the researchers document the vaccine administered?

v. What methods were used to estimate gestational age at time of maternal vaccination?

The concept of interest is the use of RWD to produce RWE to estimate the effect of immunization during pregnancy on the infant. The review considered any studies of women immunized during pregnancy. Studies of infant immunizations were excluded. Studies reporting on effectiveness or safety outcomes in the infant were included. Studies of

Vaccine. Author manuscript; available in PMC 2021 October 08. 
pregnant women and their infants were included (human studies only). Participants were from any population, reproductive age, and with any health status.

Studies with effectiveness end points describing the occurrence of vaccine-preventable disease (influenza or pertussis) in infants born to immunized mothers were included and collected as an open-ended variable in order to capture maximum data on how effectiveness end points have been defined and measured. Studies with safety end points describing adverse events in infants born to immunized mothers were included. This concept was collected as an open-ended variable in order to capture the full range of safety end points considered in the literature. Studies conducted in any country were included, as there were no restrictions on cultural factors, geographic location or specific settings. Studies using RWD (as defined by the FDA framework) such as electronic health records, administrative claims, pharmacy benefit records, or registries were included. Letters, reviews (except to search references of systematic reviews), case reports, and animal studies were excluded. Articles published in English were included. Articles published during the 10 years prior to the search were included. The search was limited to the previous 10 years to include the time period in which maternal immunizations have been administered to pregnant women for the purpose of conferring immunity on the infant.

\subsection{Search strategy}

The search strategy aimed to locate published primary studies (rather than reviews or commentaries upon studies). A preliminary search of MEDLINE and Embase was undertaken to identify articles on the topic. A librarian developed search strategies to optimize sensitivity and specificity of the electronic search. A preliminary search for existing scoping reviews on the topic was conducted using the search terms "vaccine," "vaccinations," and "scoping review." The search was conducted in PubMed, Embase and the JBI Database of Systematic Reviews and Implementation Reports. The text words contained in the titles and abstracts of identified articles, and the index terms used to describe the articles were analyzed by a reference librarian [21] to develop a full search strategy for PubMed and for Embase (Appendix A). The search was conducted on August 8 , 2019. The reference lists of systematic reviews identified in the electronic search were searched to identify articles missed by the electronic search. Scientists working on maternal vaccines at FDA were queried for additional references missed by the previous searches. Unpublished studies were not included due to the confidential nature of these studies submitted to FDA.

\subsection{Study selection}

Citations were managed using Endnote X9 (Clarivate Analytics, PA, USA). Citations from electronic searches were combined and the duplicates removed. Study selection took place in two steps: titles and abstracts were screened for general relevance, and full-text articles were screened for more detailed inclusion criteria. Two reviewers (TL, JM) conducted each level of screening using a data collection form, and a third expert was available for adjudication of discrepancies. At first screening, articles were included for full-text review if either reviewer recommended inclusion; at full-text screening agreement between the two reviewers was required for inclusion. If the two reviewers disagreed on inclusion of the 
citation, a third reviewer adjudicated. Studies that did not meet the inclusion criteria were excluded and reasons for exclusion were reported using a PRISMA-ScR flow diagram [23].

\subsection{Data extraction}

Data were extracted from papers included in the scoping review by two independent reviewers using a data extraction tool developed by the authors. The data extracted included specific details about the key elements in a spreadsheet format. The items that were extracted are listed in Appendix B. The draft data extraction tool was modified and revised as necessary during the process of extracting data from each included paper. Disagreements that arose between the reviewers were resolved through discussion, or with a third reviewer. Extraction errors were minimized by cross-checking results, the use of a standardized data extraction form, and by piloting the data extraction form with reviewers.

\subsection{Data presentation}

Quantitative analysis was limited to descriptions of the numbers and percentages of studies addressing a specific issue. Quality assessments and risk of bias assessments were not performed, as the aim of the scoping review was to provide an overview of the map of evidence rather than to answer a particular question $[23,25]$.

\section{Results}

The searches of PubMed and Embase were conducted in August 2019 (and updated in October 2020) and yielded 584 citations (Fig. 1). An additional 20 citations were identified through hand searches of references cited in systematic reviews, queries to colleagues, and monthly updates to the electronic searches. After removing duplicates, 482 records went through title and abstract screening. Of these, 147 received full text screening and 48 studies were eligible for inclusion. After full text screening, studies were excluded for the following reasons: 11 were not original studies; 12 did not report on immunization of pregnant women with influenza or pertussis; 7 did not report on vaccine safety or effectiveness outcomes in the infant; and 69 did not report on data collected in EHR or administrative claims or registries.

\subsection{Studies using RWD to measure pediatric safety and effectiveness of influenza or pertussis vaccines administered to the mother during pregnancy}

Forty-eight studies met the study criteria and are listed in Table 1. Their characteristics are summarized in Table 2 (Supplementary materials). Most of the studies (31 [64.6\%]) reported on influenza vaccines, 14 (29.2\%) reported on pertussis vaccines, and 3 studies $(6.3 \%)$ reported on both influenza and pertussis vaccines. It was not a purpose of this review to contrast studies of influenza vaccines with studies of pertussis vaccines and we did not test for statistical differences in key characteristics. After noting general similarities (study design type, countries where study was conducted, percent reporting on safety, percent reporting on effectiveness) in studies stratified by vaccine type, the review continued without stratification by vaccine type. Thirty-seven studies (77.1\%) reported on safety outcomes, 11 (22.9\%) reported on effectiveness outcomes. Of the studies reporting on safety outcomes, 33 reported on pregnancy outcomes such as stillbirth, fetal death, low birth weight, preterm 
delivery and related outcomes. The remaining 4 studies reported on morbidity and mortality in infancy or childhood, including one study that examined autism in childhood [26-29]. Of the studies reporting on effectiveness outcomes, 3 reported on laboratory confirmed pertussis and two studies reported on laboratory confirmed influenza as study outcomes [30-34]. Six studies reported on endpoints that included a range of respiratory conditions, influenza-like symptoms, hospitalizations, health care encounters and all-cause mortality [35-40].

\subsection{Electronic healthcare, administrative claims, or patient registry databases used in the eligible studies}

Many studies (22 or $45.8 \%$ ) used more than one database or data source, and more than one type of data. As an example, Beau and colleagues (2014) reported using the following databases in their study in France: EFEMERIS (Evaluation chez la Femme Enceinte des MEdicaments et de leurs RISques), combining information from five data sources: French Health Insurance System (Caisse Primaire d'Assurance Maladie, CPAM), Mother and Child Protection Center (Protection Maternelle et Infantile, PMI), the Antenatal Diagnosis Center (Centre de Diagnostic Anténatal; CDA), the French hospital medical information system (Programme de médicalisation des systèmes d'information; PMSI); The French National Health Insurance Fund for Salaried Workers (Caisse National de l'Assurance Maladie des Travailleurs Salariés; CNAMTS), and recorded vaccination data [41]. Regan and colleagues (2016) reported using multiple databases in Western Australia: Midwives Notification system, Western Australia Antenatal Influenza Vaccination Database, Western Australia Department of Health's Hospital Morbidity Database Collection, Western Australia Notifiable Infectious Diseases Database, Death Registrations database [38]. Another example can be found in the ten studies that reported using variations of the Vaccine Safety Datalink, a collaboration between the US Centers for Disease Control and Prevention and nine health care organizations [42]. While ten studies reported using the VSD as a data source, one study reported using 8 of the VSD sites, five reported using seven sites, two reported using six sites, one reported using five sites and two sites, each [40,43-51]. It was not possible to determine from the publication whether studies used the same sites. Forty-one studies ( $85.4 \%$ ) reported use of at least one electronic health record database, 25 $(52.1 \%)$ reported use of administrative claims, and 21 (43.8\%) reported use of registries.

\subsection{Methods used to link maternal and infant records}

Forty-two (87.5\%) of the studies reported linking data from maternal and infant data sources. Of these, 16 relied on unique identifiers (deterministic methods) to link the records (national identifiers as in the French, Swedish or Taiwanese systems, or system specific identifiers as in the Parkland Hospital prenatal system or the Kaiser Permanente systems). In six studies (12.5\%), a range of methods were used to link maternal and infant records including two using probabilistic methods, two using combined deterministic and probabilistic methods and two reporting methods that included queries to the mothers' physicians $[29,32,34,36,38,52]$. The two publications reporting use of probabilistic data linkage reported on different outcomes of the same study (stillbirths in one publication, and respiratory infections in the second publication) $[38,52]$. They provided minimal information about methods used for data linkage (i.e. "As full name and date of birth were the only variables available across all data sources and variations in the quality of these variables 
were possible, probabilistic matching was used) [38]. Only four studies (8.3\%) reported on the percent of successful linkages, which ranged from $62.6 \%$ to $98.8 \%[29,35,36,39]$. The low percentage of studies reporting details of data linkages is despite reporting guidelines to the contrary [53]. Twenty-two studies (45.8\%) did not report the methods used for linking maternal and infant records. In six studies (12.5\%), maternal-infant record linkage was not used and the study outcomes, such as fetal death, spontaneous abortion, and preterm delivery, were obtained from the maternal record.

\subsection{Documentation of vaccine administered}

Eight studies (16.7\%) reported on the coding system used to identify vaccines. These included CVX codes (the Centers for Disease Control and Prevention's vaccine administered code set), database specific codes, HL7 codes (another way of describing CVX codes), and other combinations of coding systems. In another two studies (4.2\%), the researchers contacted the provider to obtain information about the specific vaccine administered. An additional five studies (10.4\%) were conducted in settings where a single vaccine product was administered and there was no further need to rely on coding to document the vaccine product. The majority of studies (33 or 68.8\%) did not report any details about documenting the specific vaccine product administered (manufacturer and vaccine product name).

\subsection{Methods and sources of information about gestational age}

Forty-two studies (87.5\%) referenced gestational age in a variety of contexts (i.e. gestational age at delivery or gestational age at immunization) and gestational age was measured in weeks, trimesters, or broad categories (e.g. preterm vs term). Eleven studies (22.9\%) used fields such as last menstrual period, ultrasound, or due date from the maternal medical record to estimate gestational age. Four studies (8.3\%) reported using fields from the infant medical record or birth certificate that reflected the infant's gestational age at birth and two studies (4.2\%) reported using algorithms or codes without detailing the source of the data. Twenty-five studies $(52.1 \%)$ did not report methods used to calculate gestational age or referred the reader to related publications for further information.

\section{Discussion}

We identified forty-eight studies that met the inclusion criteria of the scoping review protocol. These used RWD/RWE to assess safety or effectiveness of influenza or pertussis vaccinations administered to pregnant women with respect to pregnancy, infant or child outcomes. They provide a resource with which it is possible to identify practices used and potential areas of improvement. After answering the first question (What studies using RWD have been conducted to measure pediatric safety and effectiveness of influenza or pertussis vaccines administered to the mother during pregnancy?), limited information was retrieved regarding questions ii through $\mathrm{v}$. What appeared to be a simple question (the number of databases or data sources used) proved to elude simple categorization. Some authors listed their data sources by naming each database (e.g.., Beau et al 2014, Fell et al 2016), others used global terms (i.e. "electronic health data", "a centralized medical record charting system") to describe their data source (i.e., Kharbanda et al 2017, Morgan et al 2015) and still others provided no specific information (e.g., Fabiani et al 2015 
reported "different regional databases and registries") [36,41,47,54,55]. It proved difficult to categorize databases as claims, electronic medical records, or registries because of differences in terminology (the Nordic countries using the word "registry" more broadly than it is used elsewhere).

The majority of studies reported linkage of at least two data sources (maternal and infant). Of these, a majority did not report any information about methods used to link maternal and infant data, and only four reported the percentage matched successfully. Reporting fell short of the details suggested by Pratt and colleagues in their checklist for reporting key elements of the linkage process [56]. Of note, no authors assessed the validity of the linkages, and no authors assessed the potential for bias arising from the linkage process. These omissions have bearing on the internal and external validity of the studies, as well as their reproducibility. It is also of interest to note the large number of studies conducted in data systems with unique identifiers used for deterministic linkage, where validity and reproducibility of the linkages may be more reliable. This often occurs in countries with registries dedicated to assessment of pregnancy exposures and outcomes. Without addressing the validity of deterministic vs probabilistic methods, it is questionable whether this body of research has resulted from expediency and convenience regarding linkage of maternal and infant data. This leads to the question of feasibility of conducting valid maternal-infant record linkage in other populations such as the Medicaid population as demonstrated by Knox et al (2019) or in other claims based systems [57]. The current body of studies using RWD/RWE to describe immunization of pregnant women and its effects on the infant may not be generalizable to the larger population of pregnant women because of non-representativeness related to the data sources used to generate these studies. It also raises the issue that different research models using RWD/RWE may be available when using registries or when using claims, electronic health records, or other combinations of these different types of data sources.

A small number of studies reported on methods of ascertaining the specific vaccine product administered, limiting reproducibility and our ability to interpret study results. Documentation of the vaccine administered will become increasingly important in studies using RWD/RWE as multiple vaccines for COVID-19 enter practice, and intense scrutiny of such studies will increase the value of detailed reporting of methods used to document the vaccine product [20]. Several of the studies identified in this scoping review were conducted in environments where only one product was administered, while others relied on time consuming methods, such as inquiries to the woman's physician. It is desirable that researchers and stakeholders develop methods to accurately and completely characterize the vaccine product, for example, by collaborating to develop coding solutions that differentiate between products, and agreeing on the level of detail required (i.e. dosage, lot number and other characteristics).

Gestational age is an important variable in this area of research because of the desirability of documenting the time of exposure (gestational age at time of immunization) and its importance in defining important outcomes such as stillbirth, spontaneous abortion, preterm birth, and small for gestational age birth. Even when the immunization date was known, studies varied in methods of assigning gestational age to the date, with the majority not 
reporting their methods at all. Six studies did not report any information about gestational age. This appears to be an area where research methods can be further developed to reach agreement on the level of precision (e.g., whether trimester of exposure is adequately precise) and the best way to measure gestational age in electronic medical records or in claims. It would also be helpful to follow the reporting guidelines for key variables and describe in the supplementary materials the algorithm used to calculate gestational age, following guidelines for reporting of key variables in pharmacoepidemiology [53].

A strength of this scoping review is that it followed best methodologic practices to map studies using real-world data (RWD) to measure pediatric safety and effectiveness of vaccines administered to pregnant women. It followed an a priori, published protocol, using two reviewers for screening and extraction, and hand searches of systematic review references [22]. A limitation may be that a number of publications overlapped in their use of data sources and time periods, however this reflects the landscape of the published literature and provides a context for understanding current practices. Additionally, the search strategy did not include terms for "autism" and may have missed studies assessing the relationship between vaccination and autism. The scoping review was limited to the questions described earlier in this paper and did not characterize the types of outcomes studied using RWD. Future systematic reviews can contribute greatly by describing more details about the different outcomes (including long term outcomes) that can be studied using RWD/RWE. Another limitation is that while we assessed reporting of maternal-infant data linkages, we did not assess reporting of other types of data linkages. Given the many studies using multiple data sources it would be of interest to learn more about the various data linkages that were used.

\section{Conclusion}

The use of RWD/RWE to study safety and effectiveness of vaccination of pregnant women with respect to pregnancy, infant and child outcomes has increased in its importance since the time that this scoping review was proposed because COVID-19 vaccine trials have not initially enrolled and completed studies with pregnant women. The issues that have been identified may also be relevant to efforts to use RWD/RWE to describe pediatric safety and effectiveness of therapeutics administered to pregnant women, although the issue of therapeutics was outside of the scope of the present review. The evidence accrued in this review has an increased urgency, and it is hoped that researchers planning to use RWD/RWE to study vaccines in pregnant women will follow the reporting guidelines as outlined in RECORD-PE or in STaRT-RWE [53,58]. This current scoping review provides an overview of the evidence base about studies using RWD/RWE to assess effects of maternal vaccination on safety and effectiveness in infants. It is timely and actionable in a way not anticipated, for planning studies using RWD/RWE to study vaccines against COVID-19 in pregnant women and potential effects in their infants.

\section{Ethics}

The scoping review methodology synthesizes information from publicly available publications and is not considered human subject research. 


\section{Supplementary Material}

Refer to Web version on PubMed Central for supplementary material.

\section{Acknowledgments}

Amy Abernethy, MD, PhD, Principal Deputy Commissioner (former), for her encouragement and support of this project.

The FDA Perinatal Health Center of Excellence for its award program fostering FDA wide perinatal and maternal health research.

Lee Bernstein, lead librarian, US Food and Drug Administration, Office of the Commissioner, for developing the search strategies.

Jane Mutanga, $\mathrm{PhD}$, for data extraction.

Funding

This scoping review has been conducted as part of a project "Database attributes for assessing pediatric safety and effectiveness of vaccines administered to the mother during pregnancy" funded by FDA's Perinatal Health Center of Excellence (PHCE) Intramural Funding Program. The funders have no input in developing the protocol or conducting the review. Funding supported a post-doctoral fellow (JM) who assisted in screening and data extraction.

\section{Appendix A:: Search strategies}

PubMed

\#1: “Pertussis Vaccine”[Majr]OR “Influenza Vaccines”[Majr]

\#2: "Infant”[Mesh] OR infant[TIAB]

\#3: "Pregnancy”[Mesh]

\#4: \#1 AND \#2 AND \#3

\#5: “Treatment Outcome”[Mesh]

\#6: efficacy[TIAB] OR effective*[TIAB] or safety OR risk

\#7: \#5 OR \#6

\#8: \#4 AND \#7

\#9: “Case Reports” [Publication Type] OR “Editorial” [Publication Type]

\#10: \#8 NOT \#9= 241

\#11: pertussis vaccin* OR pertussis immuni* OR influenza vaccin* OR influenza immuni*

\#12: pregnancy[TIAB] OR maternal[TIAB] OR pregnant[TIAB]

\#13: \#11 AND \#12 AND \#7 = 87

\#14: \#10 AND \#13 = 328 
\#15: \#14, Humans only = 241

Embase

English; last 10 years

\#1: 'pertussis vaccine’/exp/mj OR 'influenza vaccine’/exp/mj

\#2: 'pregnancy' OR maternal OR pregnant

\#3: 'infant'

\#4: 'treatment outcome' OR effectiv* OR efficacy OR safety OR risk

\#5: \#1 AND \#2 AND \#3 and \#4

\#6: 'case report'/exp OR ‘editorial'/exp ‘/exp

\#7: \#5 NOT \#6 = 184

\section{Appendix B:: Charting/extraction fields}

Key information that was charted/extracted:
a. Author(s)
b. Year of publication
c. Country(ies) where the study was conducted
d. Electronic healthcare database(s), administrative claims or patient registry databases used as source of study data
e. Type of electronic healthcare database(s), administrative claims or patient registry databases used as source of study data
f. Number of pregnant women in study (if not reported, number of infants in the study)
g. Vaccines studied (influenza, pertussis or both)
h. Coding system for describing vaccine administered (for example, CVX, ICD-9, ICD-10, HCPCS, other, none)
i. Measurement of gestational age
j. Safety outcomes
k. Effectiveness outcomes
I. Method of mother-infant data linkage (if used) 


\section{Appendix C.: Supplementary material}

Supplementary data to this article can be found online at https://doi.org/10.1016/

j.vaccine.2021.05.071.

\section{References}

[1]. Omer SB. Maternal Immunization. New Engl J Med 2017;376(13):1256-67. [PubMed: 28355514]

[2]. Abu Raya B, Edwards KM, Scheifele DW, Halperin SA. Pertussis and influenza immunisation during pregnancy: a landscape review. Lancet Infect Dis 2017;17(7):e209-22. [PubMed: 28433704]

[3]. Bergin N, Murtagh J, Philip RK. Maternal Vaccination as an Essential Component of Life-Course Immunization and Its Contribution to Preventive Neonatology. Int J Environ Res Public Health 2018;15(5).

[4]. Nunes MC, Cutland CL, Madhi SA. Influenza Vaccination during Pregnancy and Protection against Pertussis. New Engl J Med 2018;378(13):1257-8. [PubMed: 29590549]

[5]. Rasmussen SA, Watson AK, Kennedy ED, Broder KR, Jamieson DJ. Vaccines and pregnancy: past, present, and future. Seminars Fetal Neonatal Med 2014;19 (3):161-9.

[6]. Vojtek I, Dieussaert I, Doherty TM, et al. Maternal immunization: where are we now and how to move forward?. Ann Med 2018;50(3):193-208. [PubMed: 29308916]

[7]. Akinsanya-Beysolow I, Jenkins R, Meissner HC. Advisory Committee on Immunization Practices (ACIP) recommended immunization schedule for persons aged 0 through 18 years-United States, 2013. MMWR Supplements 2013;62(1):2-8. [PubMed: 23364302]

[8]. Harper SA, Fukuda K, Uyeki TM, Cox NJ, Bridges CB. Prevention and control of influenza: recommendations of the Advisory Committee on Immunization Practices (ACIP). MMWR Recommendations Rep: Morbidity Mortal Weekly Rep Recommendations Rep 2004;53(Rr-6):140.

[9]. US Department of Health and Human Services Centers for Disease Control and Prevention. Information for Adult Patients. https://www.cdc.gov/vaccines/schedules/downloads/adult/adultschedule-easy-read.pdf. Published 2018. Accessed September 28, 2018, 2018.

[10]. Updated recommendations for use of tetanus toxoid, reduced diphtheria toxoid, and acellular pertussis vaccine (Tdap) in pregnant women-Advisory Committee on Immunization Practices (ACIP), 2012. MMWR Morb Mortal Weekly Rep 2013;62(7):131-135.

[11]. Moriarty LF. Infants and the seasonal influenza vaccine: A global perspective on safety, effectiveness, and alternate forms of protection. Hum Vaccines Immunother 2014;10(9):2721-8.

[12]. Furuta M, Sin J, Ng ESW, Wang K. Efficacy and safety of pertussis vaccination for pregnant women - a systematic review of randomised controlled trials and observational studies. BMC Pregnancy Childbirth 2017;17(1):390. [PubMed: 29166874]

[13]. US Department of Health and Human Services Food and Drug Administration. Framework for FDA's Real-world Evidence Program. In: US Department of Health and Human Services Food and Drug Administration; 2018.

[14]. Steinhoff MC, Omer SB, Roy E. Neonatal outcomes after influenza immunization during pregnancy: a randomized controlled trial. CMAJ: Can Med Associat J = J de l'Association medicale canadienne 2012;184(6):645-53.

[15]. Munoz FM, Bond NH, Maccato M, et al. Safety and immunogenicity of tetanus diphtheria and acellular pertussis (Tdap) immunization during pregnancy in mothers and infants: a randomized clinical trial. JAMA 2014;311(17):1760-9. [PubMed: 24794369]

[16]. Dreyer NA, Bryant A, Velentgas P. The GRACE Checklist: A Validated Assessment Tool for High Quality Observational Studies of Comparative Effectiveness. J Manag Care Spec Pharm 2016;22(10):1107-13. [PubMed: 27668559]

[17]. Berger ML, Sox H, Willke RJ, et al. Good practices for real-world data studies of treatment and/or comparative effectiveness: Recommendations from the joint ISPOR-ISPE Special Task 
Force on real-world evidence in health care decision making. Pharmacoepidemiol Drug Saf 2017;26(9):1033-9. [PubMed: 28913966]

[18]. Hall GC, Sauer B, Bourke A, Brown JS, Reynolds MW, LoCasale R. Guidelines for good database selection and use in pharmacoepidemiology research. Pharmacoepidemiol Drug Saf 2012;21(1):1-10.

[19]. Miksad RA, Abernethy AP. Harnessing the Power of Real-World Evidence (RWE): A Checklist to Ensure Regulatory-Grade Data Quality. Clin Pharmacol Ther 2018;103(2):202-5. [PubMed: 29214638]

[20]. Fell DB, Dimitris MC, Hutcheon JA, et al. Guidance for design and analysis of observational studies of fetal and newborn outcomes following COVID-19 vaccination during pregnancy. Vaccine 2021;39(14):1882-6. [PubMed: 33715900]

[21]. Peters M, Godfrey CM, McInerney P, Baldini Soares C, Khalil H, Parker D. Scoping Reviews. In: Aromataris E, Munn Z, editors. Joanna Briggs Institute Reviewer's Manual. The Joanna Briggs Institute; 2017.

[22]. Peters MDJ, Marnie C, Tricco AC, et al. Updated methodological guidance for the conduct of scoping reviews. JBI Evidence Synthesis 2020;18(10):2119-26. [PubMed: 33038124]

[23]. Tricco AC, Lillie E, Zarin W, et al. PRISMA Extension for Scoping Reviews (PRISMA-ScR): Checklist and Explanation. Ann Intern Med 2018;169 (7):467-73. [PubMed: 30178033]

[24]. Aromataris E, Joanna Munn Z. Briggs Institute Reviewer's Manual. 4th ed:. The Joanna Briggs Institute; 2017.

[25]. Munn Z, Peters MDJ, Stern C, Tufanaru C, McArthur A, Aromataris E. Systematic review or scoping review? Guidance for authors when choosing betweena systematic or scoping review approach. BMC Med Res Method 2018;18 (1):143.

[26]. Becerra-Culqui TA, Getahun D, Chiu V, Sy LS, Tseng HF. Prenatal Tetanus, Diphtheria, Acellular Pertussis Vaccination and Autism Spectrum Disorder. Pediatrics 2018;142(3).

[27]. Layton JB, Butler AM, Li D, et al. Prenatal Tdap immunization and risk of maternal and newborn adverse events. Vaccine 2017;35(33):4072-8. [PubMed: 28669620]

[28]. Ludvigsson JF, Strom P, Lundholm C, et al. Maternal vaccination against H1N1 influenza and offspring mortality: population based cohort study and sibling design. Bmj-Br Med J 2015;351.

[29]. Walsh LK, Donelle J, Dodds L, et al. Health outcomes of young children born to mothers who received 2009 pandemic H1N1 influenza vaccination during pregnancy: retrospective cohort study. BMJ 2019;366:14151. [PubMed: 31292120]

[30]. Amirthalingam G, Andrews N, Campbell H, et al. Effectiveness of maternal pertussis vaccination in England: an observational study. Lancet (London, England) 2014;384(9953):1521-8.

[31]. Baxter R, Bartlett J, Fireman B, Lewis E, Klein NP. Effectiveness of Vaccination During Pregnancy to Prevent Infant Pertussis. Pediatrics 2017;139(5).

[32]. Byrne L, Campbell H, Andrews N, Ribeiro S, Amirthalingam G. Hospitalisation of preterm infants with pertussis in the context of a maternal vaccination programme in England. Arch Dis Child 2018;103(3):224-9. [PubMed: 28814424]

[33]. Molgaard-Nielsen D, Fischer TK, Krause TG, Hviid A. Effectiveness of maternal immunization with trivalent inactivated influenza vaccine in pregnant women and their infants. J Intern Med 2019.

[34]. Walker JL, Zhao H, Dabrera G, et al. Assessment of Effectiveness of Seasonal Influenza Vaccination During Pregnancy in Preventing Influenza Infection in Infants in England, 20132014 and 2014-2015. J Infect Dis 2020;221 (1):16-20. [PubMed: 31711165]

[35]. Becker-Dreps S, Butler AM, McGrath LJ, et al. Effectiveness of Prenatal Tetanus, Diphtheria, Acellular Pertussis Vaccination in the Prevention of Infant Pertussis in the U.S. Am J Prev Med 2018;55(2):159-66. [PubMed: 29910115]

[36]. Fell DB, Wilson K, Ducharme R, et al. Infant Respiratory Outcomes Associated with Prenatal Exposure to Maternal 2009 A/H1N1 Influenza Vaccination. PLoS ONE 2016;11(8):e0160342.

[37]. Khodr ZG, Bukowinski AT, Gumbs GR, Conlin AMS. Tetanus, diphtheria, and acellular pertussis vaccination during pregnancy and reduced risk of infant acute respiratory infections. Vaccine 2017;35(42):5603-10. [PubMed: 28916245] 
[38]. Regan AK, de Klerk N, Moore HC, Omer SB, Shellam G, Effler PV. Effect of Maternal Influenza Vaccination on Hospitalization for Respiratory Infections in Newborns: A Retrospective Cohort Study. Pediatr Infect Dis J 2016;35 (10):1097-103. [PubMed: 27314823]

[39]. Shakib JH, Korgenski K, Presson AP, et al. Influenza in Infants Born to Women Vaccinated During Pregnancy. Pediatrics 2016;137(6).

[40]. Sukumaran L, McCarthy NL, Kharbanda EO, et al. Infant Hospitalizations and Mortality After Maternal Vaccination. Pediatrics 2018.

[41]. Beau AB, Hurault-Delarue C, Vidal S, et al. Pandemic A/H1N1 influenza vaccination during pregnancy: a comparative study using the EFEMERIS database. Vaccine 2014;32(11):1254-8. [PubMed: 24486369]

[42]. Centers for Disease Control and Prevention. Vaccine Safety Datalink (VSD). https://www.cdc.gov/vaccinesafety/ensuringsafety/monitoring/vsd/index. html. Published 2021. Accessed April 27, 2021; 2021.

[43]. DeSilva M, Vazquez-Benitez G, Nordin JD, et al. Maternal Tdap vaccination and risk of infant morbidity. Vaccine 2017;35(29):3655-60. [PubMed: 28552511]

[44]. Donahue JG, Kieke BA, King JP, et al. Association of spontaneous abortion with receipt of inactivated influenza vaccine containing H1N1pdm09 in 2010-11 and 2011-12. Vaccine 2017;35(40):5314-22. [PubMed: 28917295]

[45]. Irving SA, Kieke BA, Donahue JG, et al. Trivalent Inactivated Influenza Vaccine and Spontaneous Abortion. Obstet Gynecol 2013;121(1):159-65. [PubMed: 23262941]

[46]. Kharbanda EO, Vazquez-Benitez G, Lipkind HS, et al. Evaluation of the association of maternal pertussis vaccination with obstetric events and birth outcomes. JAMA 2014;312(18):1897-904. [PubMed: 25387187]

[47]. Kharbanda EO, Vazquez-Benitez G, Romitti PA, et al. First Trimester Influenza Vaccination and Risks for Major Structural Birth Defects in Offspring. J Pediatr 2017;187:234-239.e234. [PubMed: 28550954]

[48]. Nordin JD, Kharbanda EO, Vazquez Benitez G, Lipkind H, Vellozzi C, Destefano F. Maternal influenza vaccine and risks for preterm or small for gestational age birth. J Pediatr 2014;164(5):1051-1057.e1052. [PubMed: 24582484]

[49]. Sukumaran L, McCarthy NL, Kharbanda EO, et al. Association of Tdap Vaccination With Acute Events and Adverse Birth Outcomes Among Pregnant Women With Prior Tetanus-Containing Immunizations. JAMA 2015;314(15):1581-7. [PubMed: 26501534]

[50]. Sukumaran L, McCarthy NL, Kharbanda EO, et al. Safety of Tetanus Toxoid, Reduced Diphtheria Toxoid, and Acellular Pertussis and Influenza Vaccinations in Pregnancy. Obstet Gynecol 2015;126(5):1069-74. [PubMed: 26444109]

[51]. Vazquez-Benitez G, Kharbanda EO, Naleway AL, et al. Risk of Preterm or SmallforGestational-Age Birth After Influenza Vaccination During Pregnancy: Caveats When Conducting Retrospective Observational Studies. Am J Epidemiol 2016;184(3):176-86. [PubMed: 27449414]

[52]. Regan AK, Moore HC, de Klerk N, et al. Seasonal Trivalent Influenza Vaccination During Pregnancy and the Incidence of Stillbirth: Population-Based Retrospective Cohort Study. Clin Infect Dis 2016;62(10):1221-7. [PubMed: 27033634]

[53]. Langan SM, Schmidt SA, Wing K, et al. The reporting of studies conducted using observational routinely collected health data statement for pharmacoepidemiology (RECORD-PE). BMJ 2018;363:k3532.

[54]. Fabiani M, Bella A, Rota MC, et al. A/H1N1 pandemic influenza vaccination:A retrospective evaluation of adverse maternal, fetal and neonatal outcomes ina cohort of pregnant women in Italy. Vaccine 2015;33(19):2240-7. [PubMed: 25820060]

[55]. Morgan JL, Baggari SR, McIntire DD, Sheffield JS. Pregnancy Outcomes After Antepartum Tetanus, Diphtheria, and Acellular Pertussis Vaccination. Obstet Gynecol 2015;125(6):1433-8. [PubMed: 26000515]

[56]. Pratt NL, Mack CD, Meyer AM, et al. Data linkage in pharmacoepidemiology:A call for rigorous evaluation and reporting. Pharmacoepidemiol Drug Saf 2020;29(1):9-17. [PubMed: 31736248] 
[57]. Knox CA, Hampp C, Palmsten K, et al. Validation of mother-infant linkage using Medicaid Case ID variable within the Medicaid Analytic eXtract (MAX) database. Pharmacoepidemiol Drug Saf 2019;28(9):1222-30. [PubMed: 31286606]

[58]. Wang SV, Pinheiro S, Hua W, et al. STaRT-RWE: structured template for planning and reporting on the implementation of real world evidence studies. BMJ 2021;372:m4856. 
Records identified through database searching (PubMed and Embase)

$(n=584)$
Additional records identified through other sources (hand searches, queries to colleagues, updates)

$(n=20)$

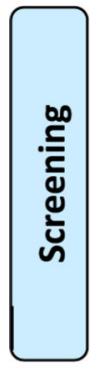

Records after duplicates removed

$$
(n=482)
$$

Studies included in Full-text articles excluded $(n=99)$

Full-text articles assessed for eligibility $(n=147)$ qualitative synthesis $(n=48)$

Fig. 1.

Flow Diagram. 


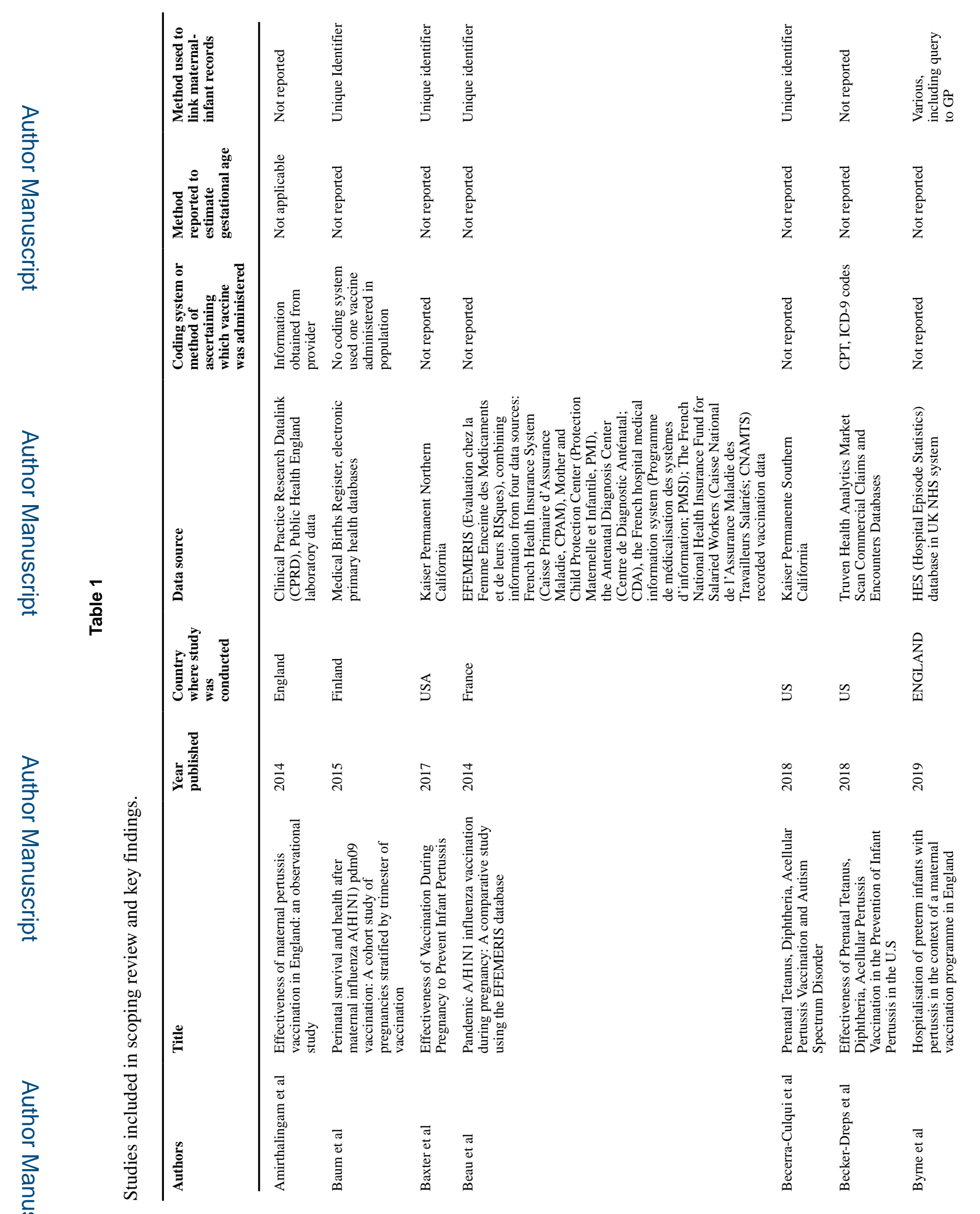

Vaccine. Author manuscript; available in PMC 2021 October 08. 


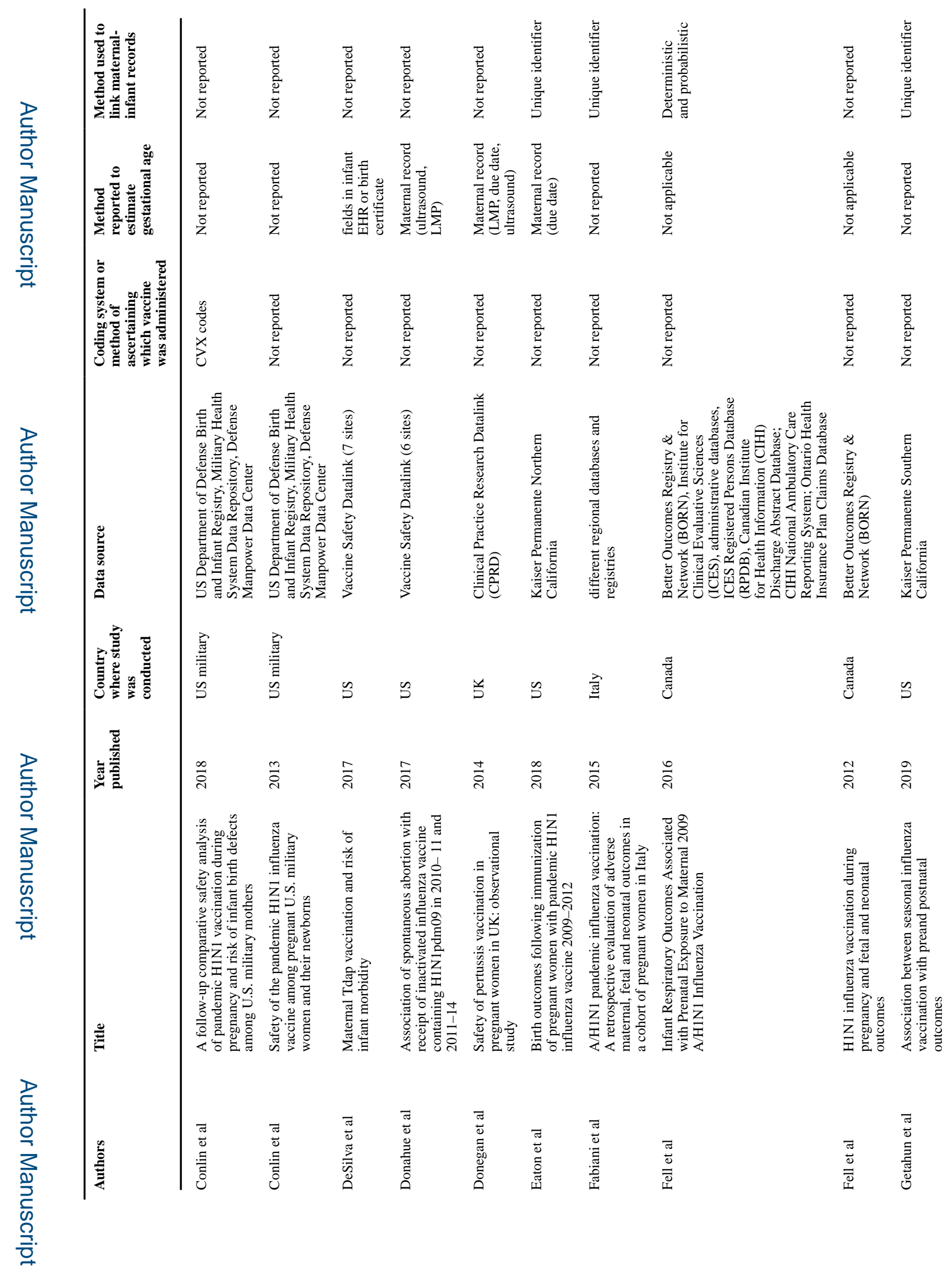

Vaccine. Author manuscript; available in PMC 2021 October 08. 


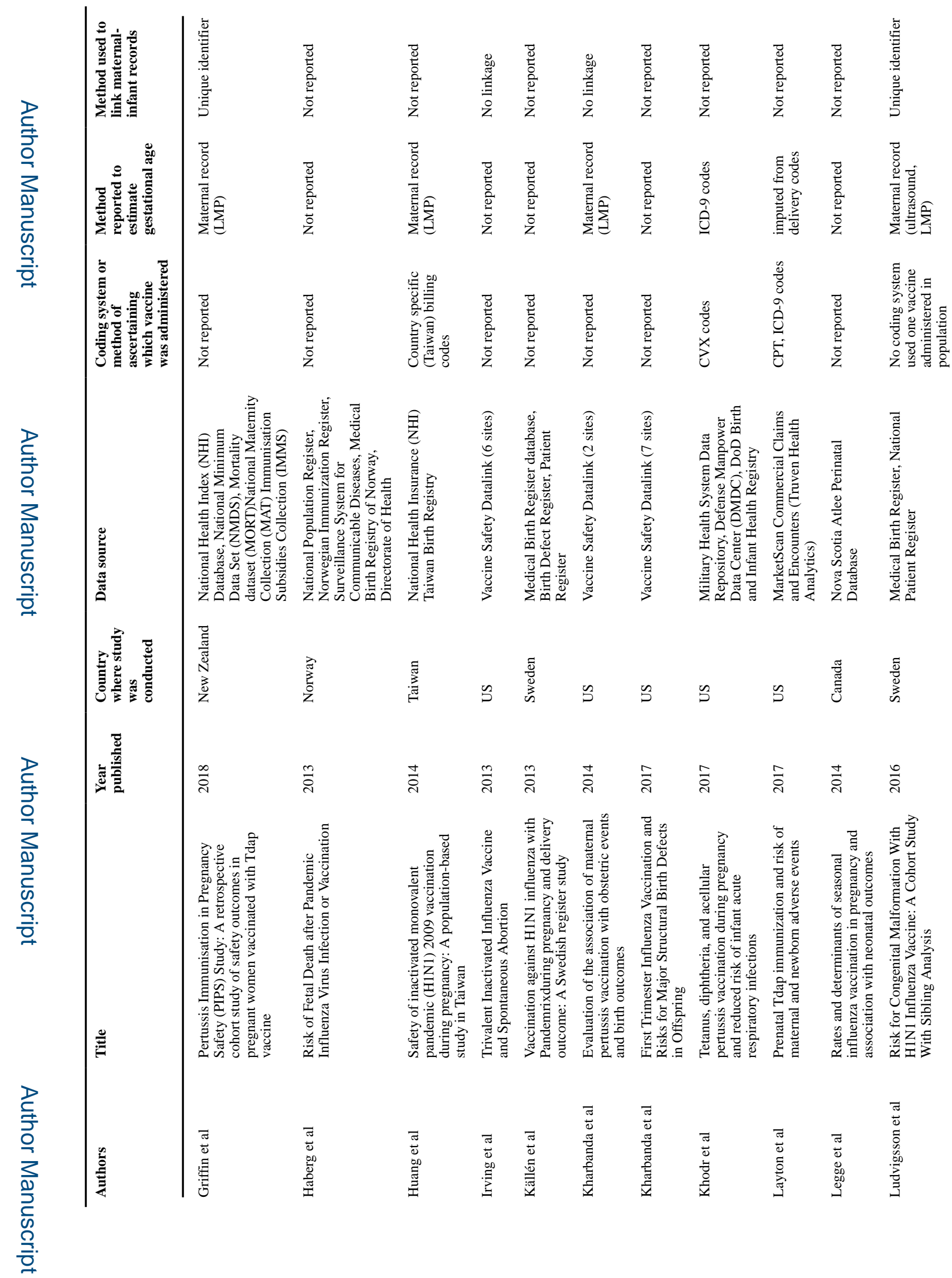

Vaccine. Author manuscript; available in PMC 2021 October 08. 


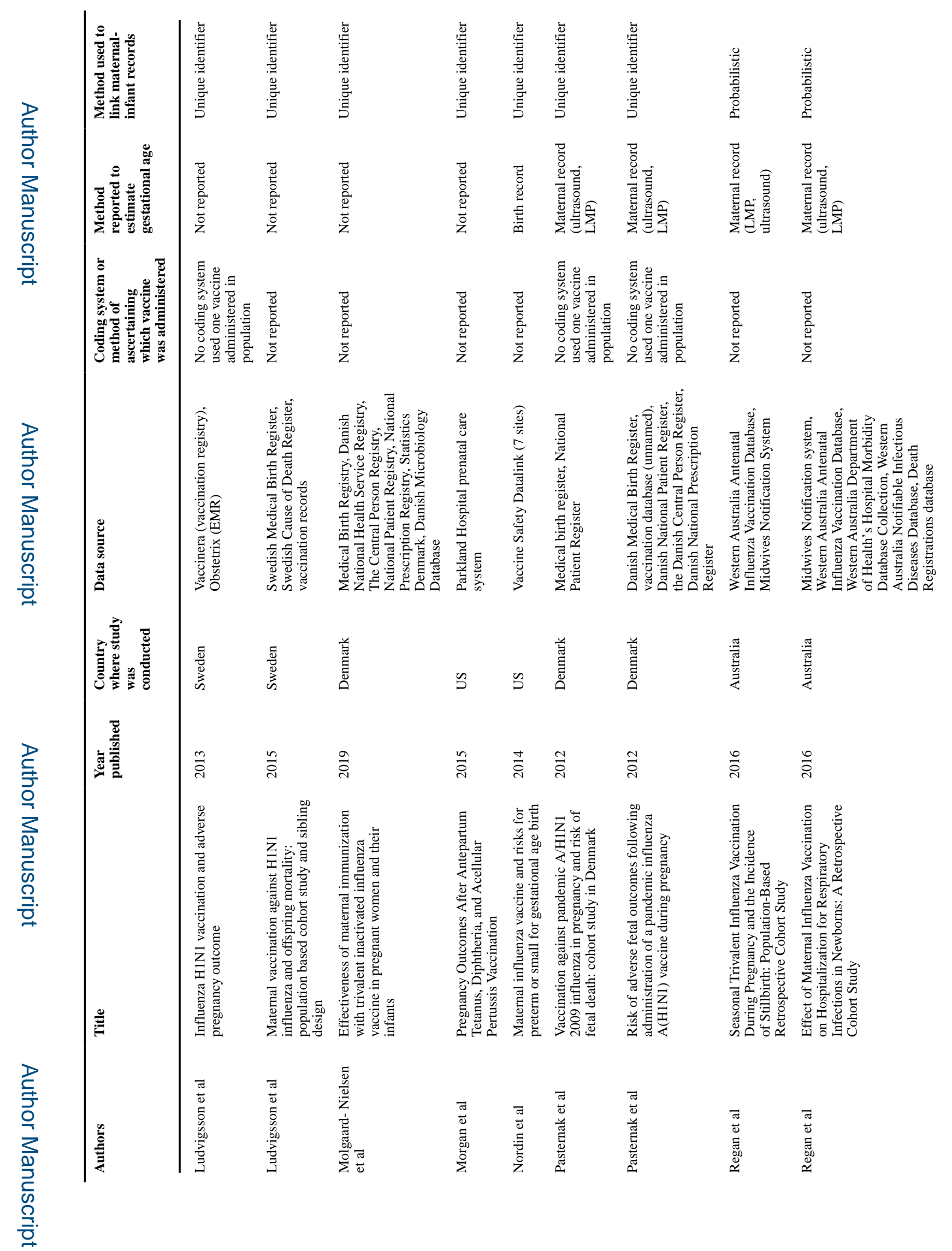

Vaccine. Author manuscript; available in PMC 2021 October 08. 


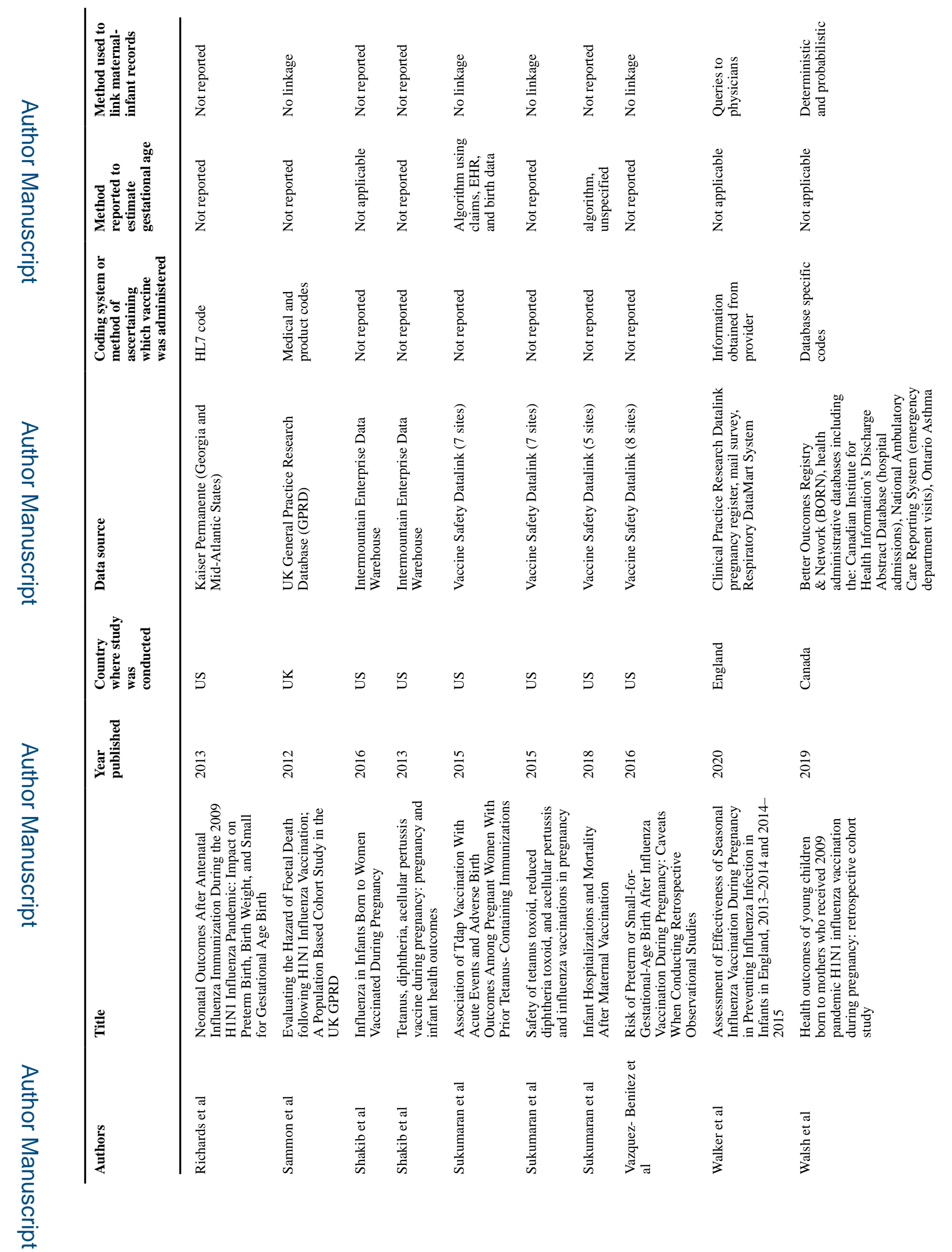

Vaccine. Author manuscript; available in PMC 2021 October 08. 
Lasky et al.

Page 22

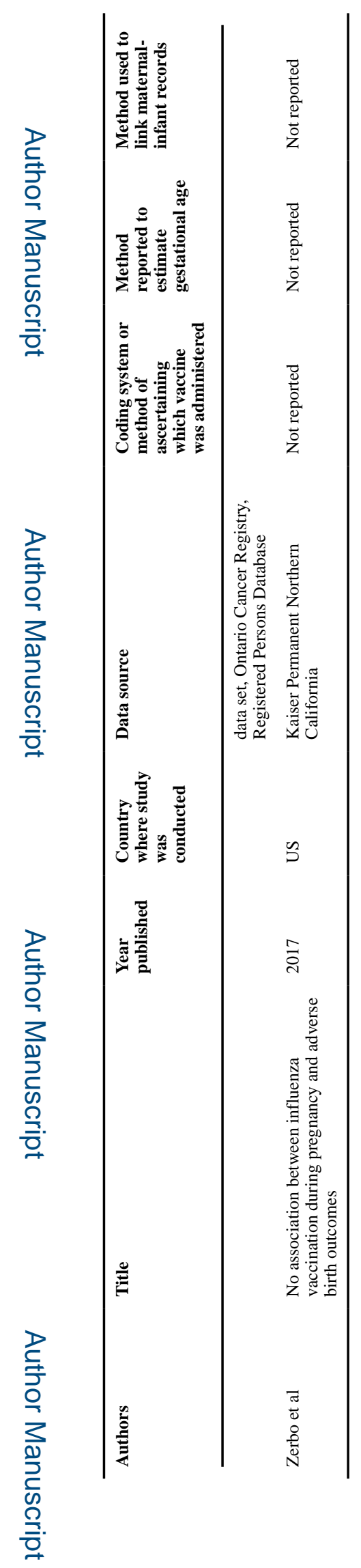

Vaccine. Author manuscript; available in PMC 2021 October 08. 